\title{
Survival in endometrial cancer in relation to minimally invasive surgery or open surgery - a Swedish Gynecologic Cancer Group (SweGCG) study
}

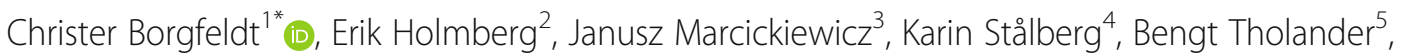 \\ Elisabeth Åvall Lundqvist ${ }^{6}$, Angelique Flöter-Rådestad ${ }^{7}$, Maria Bjurberg ${ }^{8}$, Pernilla Dahm-Kähler ${ }^{9}$, Kristina Hellman ${ }^{10}$, \\ Elisabet Hjerpe ${ }^{11}$, Preben Kjölhede ${ }^{12}$, Per Rosenberg ${ }^{6}$ and Thomas Högberg ${ }^{13}$
}

\begin{abstract}
Background: The aim of this study was to analyze overall survival in endometrial cancer patients' FIGO stages I-III in relation to surgical approach; minimally invasive (MIS) or open surgery (laparotomy).

Methods: A population-based retrospective study of 7275 endometrial cancer patients included in the Swedish Quality Registry for Gynecologic Cancer diagnosed from 2010 to 2018. Cox proportional hazard models were used in univariable and multivariable survival analyses.

Results: In univariable analysis open surgery was associated with worse overall survival compared with MIS hazard ratio, HR, 1.39 (95\% Cl 1.18-1.63) while in the multivariable analysis, surgical approach (MIS vs open surgery) was not associated with overall survival after adjustment for known risk factors (HR 1.12, 95\% Cl 0.95-1.32). Higher FIGO stage, non-endometrioid histology, non-diploid tumors, lymphovascular space invasion and increasing age were independent risk factors for overall survival.

Conclusion: The minimal invasive or open surgical approach did not show any impact on survival for patients with endometrial cancer stages I-III when known prognostic risk factors were included in the multivariable analyses.
\end{abstract}

Keywords: Endometrial cancer, Minimally invasive surgery, Survival, Risk factors

\section{Synopsis highlights}

The minimal invasive or open surgical approach did not show any impact on survival for patients with endometrial cancer stages I-III when adjusting for FIGO stage, morphology, ploidy, lymphovascular space involvement and age.

Independent risk factors for overall survival were higher FIGO stage, non-endometrioid histology, non-

\footnotetext{
* Correspondence: christer.borgfeldt@med.lu.se

'Department of Obstetrics and Gynecology, Skåne University Hospital, Lund University, SE-22185 Lund, Sweden

Full list of author information is available at the end of the article
}

diploid tumors, lymphovascular space invasion and increasing age.

\section{Background}

Endometrial cancer is the most common gynecologic cancer in developed countries. The median age at onset is around 70 years and very few women are affected before the age of 50 . There is strong evidence that the rising incidence seen throughout the Western world is associated with lifestyle factors, such as obesity, diabetes mellitus, late menopause, and an aging population $[1,2]$. The treatment for presumed early-stage endometrial

C C The Author(s). 2021 Open Access This article is licensed under a Creative Commons Attribution 4.0 International License, which permits use, sharing, adaptation, distribution and reproduction in any medium or format, as long as you give appropriate credit to the original author(s) and the source, provide a link to the Creative Commons licence, and indicate if changes were made. The images or other third party material in this article are included in the article's Creative Commons licence, unless indicated otherwise in a credit line to the material. If material is not included in the article's Creative Commons licence and your intended use is not permitted by statutory regulation or exceeds the permitted use, you will need to obtain permission directly from the copyright holder. To view a copy of this licence, visit http://creativecommons.org/licenses/by/4.0/ The Creative Commons Public Domain Dedication waiver (http://creativecommons.org/publicdomain/zero/1.0/) applies to the data made available in this article, unless otherwise stated in a credit line to the data. 
cancer involves surgery removing the uterus and performing a bilateral salpingo-oophorectomy with or without lymphadenectomy followed by radiotherapy and/or chemotherapy in selected cases based upon the estimated risk for recurrence or death. Minimally invasive surgery (MIS) in patients with endometrial cancer reduces morbidity, the time needed to resume normal activities of daily living, the number of days before return to work, length of hospital stay, and blood loss in patients with and without lymph node dissection, especially in elderly and overweight patients [3-6]. Randomized trials have shown that total laparoscopic hysterectomy seems to be equally safe as total abdominal hysterectomy, but population-based studies are needed to confirm these results [7, 8]. After the randomized controlled "LACC trial" and registry studies in cervical cancer were published, the MIS approach in cervical cancer has been disapproved in several countries, and in most national guidelines open surgery is recommended $[9,10]$. Concerning long-term survival outcomes after MIS in endometrial cancer patients' further studies are needed. The aim of this study was to analyze overall survival in patients with endometrial cancer surgical FIGO stage I-III in the whole Swedish population in relation to surgical approach, MIS vs open surgery (laparotomy) adjusting in multivariable analyses for known prognostic factors.

\section{Methods}

Reporting to the Swedish National Cancer Registry (NCR), which started in 1958, is mandatory for both pathologists and clinicians, and the registry has over 95\% coverage for all malignant tumors, of which $99 \%$ are histologically verified. The Swedish Quality Registry for Gynecologic Cancer (SQRGC) started the registration of endometrial cancer in 2010. The registration is webbased and includes information on patient and tumor characteristics, treatment details and follow-up.

Reporting to the SQRGC is performed prospectively by all hospitals and clinics in the six Swedish health care regions. Quality control is continuously performed by registrars at the regional cancer centers who monitor entered data. Through the personal identification numbers allocated to all citizens in Sweden, the SQRGC continuously receives date of death from the Population Registry enabling coverage control compared to the NCR and life-long follow-up of patients. The validity of SQRGC data has been assessed, with $70-100 \%$ agreement between registered data and the original case files [11]. Every patient can choose to opt out of registration.

\section{Study population}

The SQRGC was used to identify patients with endometrial cancer (ICD-10 code C54) stage I-III diagnosed from January 1st, 2010 through December 31st, 2018.
Inclusion criteria were age at least 18 years, histologically verified primary endometrial cancer treated with MIS or open surgical approach, endometrioid-, serous-, mucinous-, clear cell carcinomas and carcinosarcoma morphology $(n=12,582)$. Exclusion criteria were endometrial cancer FIGO stage IV $(n=295)$ and sarcomas $(n=586)$. According to the Swedish National Guidelines endometrial cancer stage IV surgery shall be performed by laparotomy. The coverage between the SQRGC and the NCR was checked and showed agreement in $97-100 \%$ (personal communication). Surgical staging was performed according to the Federation Internationale de Gynecologie et d'Obstetrique (FIGO) classification from 2009 [12]. There were exclusions due to missing data on FIGO stage $(n=1378)$, missing data on risk factors $(n=$ $1475)$, no primary surgery $(n=228)$ or surgical approach $(n=1931)$. The final data set included 7275 patients.

Most of the patients in the study were treated according to the Swedish National Guidelines for Endometrial cancer from 2011 (the guidelines were updated in 2017). In the guidelines, preoperative high-risk was defined as nonendometrioid histology (serous, clear cell carcinoma or carcinosarcoma), endometrioid adenocarcinoma FIGO grade 3, or non-diploid tumors. In preoperative high-risk tumors, a lymphadenectomy of the pelvic and para-aortic regions (up to the left renal vein) was recommended in addition to hysterectomy and salpingo-oophorectomy. Preoperative evaluation of myometrial infiltration was not included as a criterion for lymphadenectomy in the national guidelines until the revised version of 2017, when also analysis of DNA-ploidy was abandoned. Postoperatively high-risk patients in FIGO stage I-II were defined as those with non-endometrioid histology or those with endometrioid histology with two or more risk factors; grade $3, \geq 50 \%$ myometrial invasion or non-diploid tumor. Patients allotted to the postoperative high-risk group in FIGO stage I-II were recommended chemotherapy \pm brachytherapy and those with positive lymph nodes (FIGO stage III) or no lymphadenectomy were offered chemotherapy \pm external radiotherapy. Women with preoperative signs of advanced disease (FIGO stage III) were surgically treated with the intension to obtain macroscopic radicality. Lymphovascular space involvement (LVSI) was not included as a high-risk parameter in either the first or in the revised versions.

Most DNA analyses were performed by flow cytometry and a minority by image cytometry $[13,14]$. Positive LVSI was defined as obvious lymphovascular space invasion identified in routine hematoxylin and eosin staining in accordance with the Swedish Society of Pathology guidelines for endometrial cancer. There is no requirement for foci to be confirmed with immunohistochemistry, only that the pathologist making the diagnosis judges the focus to be sufficiently clear to be diagnostic. 
Patients were followed until 15 March 2019 or to emigration or death, whichever came first.

The ethical review board at Gothenburg University approved the study (Dnr 814-15).

\section{Statistics}

Distributions of descriptive data in Table 1 were compared between groups using Pearson's chi-square test and Fisher's exact tests for categorical variables, and Student's $t$ test and Wilcoxon rank-sum test for continuous variables, as appropriate. The main outcome was overall survival (OS) measured from the date of diagnosis to the date of the first event of death, emigration, or end of follow-up (March 15th, 2019). OS probabilities were calculated using the Kaplan-Meier method. The Cox proportional hazard models were used in uni- and multivariable survival analyses. The multivariable analyses included type of surgery, morphology, FIGO stage, grade of the endometrioid carcinomas, ploidy, LVSI and age at diagnosis. Hazard ratios (HR) with $95 \%$ confidence intervals $(\mathrm{CI})$ were reported for 5 years follow-up period. The proportional hazard assumption was checked using Schoenfeld's residuals. When the assumption was violated (indicated in the tables) the HR was interpreted as the mean over the 5-year follow-up period. All comparisons were two-sided, and a 5\% level of significance was used. All statistical analyses were carried out with Stata/IC 16.1 for Mac (StataCorp. 2020. Stata: Release 16. Statistical Software. College Station, TX: StataCorp LLC).

\section{Results}

The median age in both the MIS and open surgery (laparotomy) groups was 69 (range 25-98) years (Table 1 ). The percentage of patients with functional status $2-4$ (WHO-status) was similar in both groups. The median follow-up time in the MIS group was 3.5 years and in the open surgery group 4.6 years.

\section{Survival analyses}

In the univariable Cox analysis, open surgery was associated with worse survival compared with MIS (HR 1.39 95\% CI 1.18-1.63) (Fig. 1a, Table 2). In the multivariable analysis including all endometrial cancer with MIS vs. open surgery, endometrioid vs. non-endometrioid tumors, FIGO stage (IA vs. IB vs. II vs. III), diploid vs. non-diploid tumors, LVSI yes vs. no, and age groups, there was no statistically significant difference between open surgery and MIS (HR 1.12 95\% CI 0.95-1.32) (Fig. $1 \mathrm{~b}$, Table 2,). Since grade is only related to endometrioid endometrial cancer, it was not included in this multivariable analysis. Non-endometrioid tumor, non-diploid tumor, LVSI, myometrial invasion (FIGO stage IA vs
IB), FIGO stage and increasing age were all independent risk factors (Table 2).

In the corresponding uni- and multivariable analysis of endometrioid endometrial cancer, where FIGO grade $1+2$ vs 3 was added and endometrioid vs. nonendometrioid tumors was omitted, there was no statistically significant difference between MIS and open surgery (Table 3). All the other included risk factors carried independent prognostic information (Table 3). In addition, surgical approach was not an independent variable among non-endometrioid carcinomas (Table 4). FIGO stage, morphology, and age groups were all independent prognostic factors (Table 4).

In node positive patients surgical approach showed no association with OS (Table 5) Finally, the analysis comparing open surgery and robotic-assisted laparoscopy and conventional laparoscopy separately, showed no association between surgical approach and OS (Table 6).

\section{Discussion}

In this large population-based registry study in endometrial cancer patients, minimally invasive and open surgery showed no overall survival difference when known prognostic factors were included in the multivariable analyses. Independent risk factors for worse overall survival were FIGO stage, non-endometrioid histology, nondiploid tumors, lymphovascular space invasion, increasing age, and, in endometrioid tumors, FIGO grade 3.

A Danish nationwide registry study showed that the overall survival among women with early-stage endometrial cancer was improved after the introduction of robot-assisted MIS, and that MIS was associated with a lower mortality rate compared with laparotomy even after adjustment for histopathological risk groups [15]. These results are in contrast with our findings. We found no impact of surgical approach on overall survival. In comparison with the Danish study, we included more prognostic factors in the regression models i.e. DNAploidy and lymphovascular space involvement. This demonstrates the importance to include known risk factors reducing bias in observational studies. Even if our study did not show any difference in overall survival, MIS has shown to be associated with reduced surgical morbidity and faster recovery, especially in overweight patients [3-6]. Although laparoscopic robot-assisted surgery has been demonstrated to have a shorter learning curve than laparoscopic surgery, several studies still indicate that approximately 50 laparoscopic robot-assisted surgery hysterectomies must be performed to gain proficiency $[16,17]$. MIS may be used as a proxy parameter in quality measurement in endometrial cancer surgery and MIS should be accomplished in more than $80 \%$ of the patients in high volume centers [18]. 
Table 1 Patients' characteristics

\begin{tabular}{|c|c|c|c|c|}
\hline Variable & $\begin{array}{l}\text { MIS } \\
N=3742\end{array}$ & $\begin{array}{l}\text { Open surgery } \\
N=3533\end{array}$ & $\begin{array}{l}\text { Total } \\
N=7275\end{array}$ & $p$ \\
\hline$\overline{\text { Age, mean (min-max) }}$ & 68.6 (25-98) & $68.5(29-97)$ & 68.5 (25-98) & $0.93^{\mathrm{a}}$ \\
\hline Age, median (25-75\%) & $69(62-76)$ & $69(62-76)$ & $69(62-76)$ & $0.78^{b}$ \\
\hline Age, years & & & & $0.95^{\mathrm{c}}$ \\
\hline $0-59$ & $718(19.2 \%)$ & $696(19.7 \%)$ & $1414(19.4 \%)$ & \\
\hline $60-69$ & $1185(31.7 \%)$ & $1110(31.4 \%)$ & $2295(31.6 \%)$ & \\
\hline 70-79 & $1261(33.7 \%)$ & $1190(33.7 \%)$ & $2451(33.7 \%)$ & \\
\hline$\geq 80$ & 578 (15.4\%) & $572(15.2 \%)$ & $1115(15.3 \%)$ & \\
\hline WHO performance status & & & & $0.002^{\circ}$ \\
\hline 0 & $2230(59.6 \%)$ & $1453(41.1 \%)$ & $3683(50.6 \%)$ & \\
\hline 1 & 789 (21.1\%) & $634(18.0 \%)$ & $1423(19.6 \%)$ & \\
\hline 2 & $175(4.7 \%)$ & $127(3.6 \%)$ & $302(4.2 \%)$ & \\
\hline 3 & $20(0.5 \%)$ & $23(0.6 \%)$ & $43(0.6 \%)$ & \\
\hline 4 & $2(0.0 \%)$ & $5(0.1 \%)$ & $7(0.1 \%)$ & \\
\hline Missing & $526(14.1 \%)$ & $1291(36.5 \%)$ & $1817(25.0 \%)$ & \\
\hline FIGO stage & & & & $<0.001^{\mathrm{C}}$ \\
\hline IA & $2481(66.3 \%)$ & $1925(54.5 \%)$ & $4406(60.6 \%)$ & \\
\hline IB & 744 (19.9\%) & $783(22.2 \%)$ & $1527(21.0 \%)$ & \\
\hline$\|$ & $249(6.6 \%)$ & $315(8.9 \%)$ & $564(7.8 \%)$ & \\
\hline III & $268(7.2 \%)$ & $510(14.4 \%)$ & $778(10.7 \%)$ & \\
\hline Morphology & & & & $<0.001^{c}$ \\
\hline Endometrioid & $3426(91.6 \%)$ & $2960(83.8 \%)$ & $6386(87.8 \%)$ & \\
\hline FIGO grade $1-2$ & $2976(86.9 \%)$ & $2281(77.1 \%)$ & $5257(82.3 \%)$ & \\
\hline FIGO grade 3 & $362(10.6 \%)$ & $573(19.4 \%)$ & $935(14.6 \%)$ & \\
\hline Missing & $88(2.6 \%)$ & $106(3.6 \%)$ & $194(3.0 \%)$ & \\
\hline Serous & 196 (5.2\%) & $299(8.5 \%)$ & $495(6.8 \%)$ & \\
\hline Clear cell & $67(1.8 \%)$ & $108(3.1 \%)$ & $175(2.4 \%)$ & \\
\hline Carcinosarcoma & $53(1.4 \%)$ & $166(4.7 \%)$ & $219(3.0 \%)$ & \\
\hline Postoperative risk groups & & & & $<0.001^{\mathrm{C}}$ \\
\hline Low & $2299(61.4 \%)$ & $1642(46.5 \%)$ & $3941(54.2 \%)$ & \\
\hline High & $1443(38.6 \%)$ & $1891(53.5 \%)$ & $3334(45.8 \%)$ & \\
\hline LVSI & & & & $<0.001^{\mathrm{C}}$ \\
\hline No & $2547(68.1 \%)$ & $2051(58.0 \%)$ & $4598(63.2 \%)$ & \\
\hline Yes & $464(12.4 \%)$ & $646(18.3 \%)$ & $1110(15.3 \%)$ & \\
\hline Missing & $731(19.5 \%)$ & $836(23.7 \%)$ & $1567(21.5 \%)$ & \\
\hline Ploidy & & & & $<0.001^{\mathrm{C}}$ \\
\hline Diploid & $2414(64.5 \%)$ & $2005(56.8 \%)$ & $4419(60.7 \%)$ & \\
\hline Non-diploid & $719(19.2 \%)$ & $947(26.8 \%)$ & $1666(22.9 \%)$ & \\
\hline Missing & $609(16.3 \%)$ & $581(16.4 \%)$ & $1190(16.4 \%)$ & \\
\hline \multicolumn{5}{|c|}{ Follow-up (years), median (25-75\%) } \\
\hline Censored & $3.7(2.0-5.4)$ & $5.1(3.2-7.1)$ & $4.3(2.4-6.1)$ & \\
\hline Death & $2.3(1.4-3.8)$ & $2.6(1.3-4.3)$ & $2.5(1.3-4.1)$ & \\
\hline All & $3.5(1.9-5.2)$ & $4.6(2.6-6.6)$ & $4.0(2.2-5.9)$ & \\
\hline
\end{tabular}

FIGO International Federtion of Gynecology and Obstetics (Federation Internationale de Gynecologie et d'Obstetrique) LVSI Lymphovascular space involvement

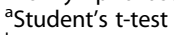

bilcoxon rank-sum test

'Pearson's chi-squared test

${ }^{d}$ Fisher's exact test 
a.

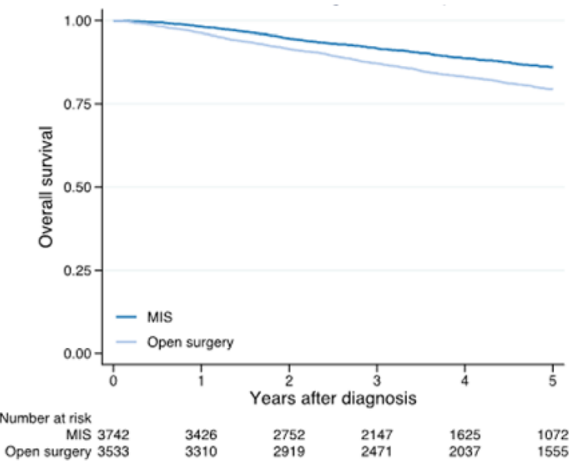

b.

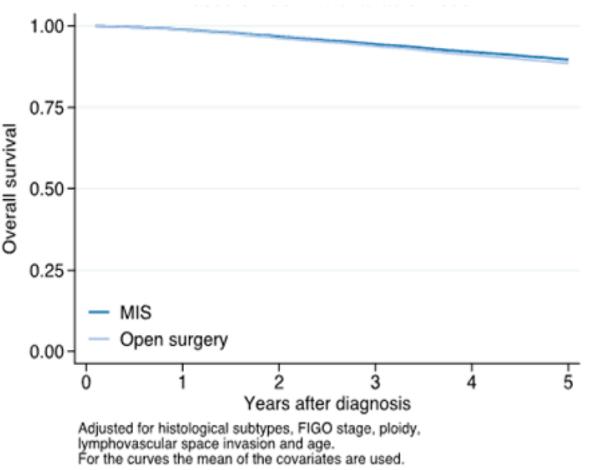

Fig. 1 a Overall survival estimates for minimally invasive surgery (MIS) (reference) and open surgery (laparotomy), hazard ratio 1.57 (95\% confidence interval 1.38-1.79) in the univariable Cox proportional hazards model. b Overall survival estimates for minimally invasive surgery (MIS) (reference) and open surgery (laparotomy) adjusted for morphology, FIGO stage, ploidy, lymphovascular space invasion, and age. Hazard ratio was non significant in the multivariable Cox proportional hazards model

Table 2 Endometrial cancer, FIGO stage I-III. Uni- and multivariable Cox proportional hazard regression analyzing overall survival including type of surgery, morphology, FIGO stage, ploidy, lymphovascular space invasion and age

\begin{tabular}{|c|c|c|c|c|c|}
\hline \multirow[t]{2}{*}{ Variables } & \multirow[t]{2}{*}{$\begin{array}{l}\text { No. of } \\
\text { patients }\end{array}$} & \multicolumn{2}{|l|}{$\begin{array}{l}\text { Univariable } \\
\text { Cox regression }\end{array}$} & \multicolumn{2}{|l|}{$\begin{array}{l}\text { Multivariable } \\
\text { Cox regression }\end{array}$} \\
\hline & & HR $(95 \% \mathrm{Cl})$ & $p$ & HR $(95 \% \mathrm{Cl})$ & $p$ \\
\hline \multicolumn{6}{|l|}{ Surgical approach } \\
\hline MIS & 2527 & Ref. & & Ref. & \\
\hline Open surgery & 2286 & $1.39(1.18-1.63)$ & $<0.001$ & $1.12(0.95-1.32)$ & 0.19 \\
\hline \multicolumn{6}{|l|}{ Endometrioid } \\
\hline Yes & 4378 & Ref. & & Ref. & \\
\hline No & 435 & $3.91(3.24-4.71)$ & $<0.001$ & $1.75(1.42-2.18)$ & $<0.001$ \\
\hline \multicolumn{6}{|l|}{ FIGO stage ${ }^{a}$} \\
\hline IA & 2978 & Ref. & & Ref. & \\
\hline IB & 1019 & $2.09(1.69-2.58)$ & $<0.001$ & $1.32(1.06-1.65)$ & 0.013 \\
\hline$\|$ & 359 & 3.07 (2.36-3.99) & $<0.001$ & $1.81(1.38-2.38)$ & $<0.001$ \\
\hline III & 457 & $6.25(5.10-7.67)$ & $<0.001$ & $2.74(2.16-3.49)$ & $<0.001$ \\
\hline \multicolumn{6}{|l|}{ Ploidy } \\
\hline Diploid & 3534 & Ref. & & Ref & \\
\hline Non-diploid & 1279 & $2.75(2.34-3.23)$ & $<0.001$ & $1.59(1.33-1.91)$ & $<0.001$ \\
\hline \multicolumn{6}{|l|}{ LVSI } \\
\hline No & 3896 & Ref. & & Ref. & \\
\hline Yes & 917 & $4.24(3.61-4.98)$ & $<0.001$ & $2.25(1.86-2.72)$ & $<0.001$ \\
\hline \multicolumn{6}{|l|}{ Age group (years) } \\
\hline $0-59$ & 943 & Ref. & & Ref. & \\
\hline $60-69$ & 1535 & $2.11(1.44-3.10)$ & $<0.001$ & $1.88(1.28-2.76)$ & 0.001 \\
\hline $70-79$ & 1624 & $4.40(3.08-6.31)$ & $<0.001$ & $3.66(2.55-5.26)$ & $<0.001$ \\
\hline 80- & 711 & $10.7(7.44-15.3)$ & $<0.001$ & $8.65(6.00-12.5)$ & $<0.001$ \\
\hline
\end{tabular}

${ }^{\mathrm{a}}$ The proportional hazard rates assumption is not fulfilled 
Table 3 Endometrioid endometrial carcinoma, FIGO stage I-III. Uni- and multivariable Cox proportional hazard regression analyzing overall survival including type of surgery, FIGO grade, FIGO stage, ploidy, lymphovascular space invasion and age

\begin{tabular}{|c|c|c|c|c|c|}
\hline \multirow[t]{2}{*}{ Variables } & \multirow[t]{2}{*}{$\begin{array}{l}\text { No. of } \\
\text { patients }\end{array}$} & \multicolumn{2}{|l|}{$\begin{array}{l}\text { Univariable } \\
\text { Cox regression }\end{array}$} & \multicolumn{2}{|l|}{$\begin{array}{l}\text { Multivariable } \\
\text { Cox regression }\end{array}$} \\
\hline & & HR $(95 \% \mathrm{Cl})$ & $p$ & HR $(95 \% \mathrm{Cl})$ & $p$ \\
\hline \multicolumn{6}{|l|}{ Surgical approach } \\
\hline MIS & 2310 & Ref. & & Ref. & \\
\hline Open surgery & 1950 & $1.33(1.10-1.61)$ & 0.003 & $1.12(0.93-1.36)$ & 0.24 \\
\hline \multicolumn{6}{|l|}{ FIGO grade ${ }^{a}$} \\
\hline $1-2$ & 3621 & Ref. & & Ref. & \\
\hline 3 & 639 & $2.47(2.01-3.05)$ & $<0.001$ & $1.31(1.02-1.66)$ & 0.031 \\
\hline \multicolumn{6}{|l|}{ FIGO stage ${ }^{a}$} \\
\hline $\mathrm{IA}$ & 2720 & Ref. & & Ref. & \\
\hline IB & 942 & $2.22(1.77-2.79)$ & $<0.001$ & $1.34(1.05-1.71)$ & 0.019 \\
\hline$\|$ & 295 & $3.36(1.70-3.28)$ & $<0.001$ & $1.55(1.10-2.18)$ & 0.012 \\
\hline III & 303 & $5.18(3.99-6.72)$ & $<0.001$ & $2.60(1.92-3.53)$ & $<0.001$ \\
\hline \multicolumn{6}{|l|}{ Ploidy } \\
\hline Diploid & 3395 & Ref. & & Ref. & \\
\hline Non-diploid & 865 & $2.01(1.64-2.45)$ & $<0.001$ & $1.43(1.15-1.78)$ & 0.001 \\
\hline \multicolumn{6}{|l|}{$\operatorname{LVSI}^{\mathrm{a}}$} \\
\hline No & 3544 & Ref. & & Ref. & \\
\hline Yes & 716 & $3.64(3.00-4.42)$ & $<0.001$ & $2.08(1.66-2.61)$ & $<0.001$ \\
\hline \multicolumn{6}{|l|}{ Age group (years) } \\
\hline $0-59$ & 886 & Ref. & & Ref. & \\
\hline $60-69$ & 1374 & $1.94(1.26-3.00)$ & 0.003 & $1.94(1.26-3.00)$ & 0.003 \\
\hline $70-79$ & 1405 & $4.10(2.73-6.17)$ & $<0.001$ & $3.82(2.54-5.76)$ & $<0.001$ \\
\hline 80- & 595 & $10.8(7.18-16.2)$ & $<0.001$ & $9.6(6.39-14.5)$ & $<0.001$ \\
\hline
\end{tabular}

A US register study including 6304 elderly (65+) middle-class women with early-stage endometrial cancer enrolled in a US national insurance program (Medicare) found a tendency towards better overall survival in favor of MIS compared with laparotomy [19]. A recent metaanalysis including six published randomized controlled trials with 3993 patients demonstrated that overall survival after MIS was similar to the overall survival after open surgery and that MIS was associated with reduced surgical morbidity, which also has been shown in a Swedish study in elderly patients $[6,20]$. Socioeconomic conditions and surgical allocation may affect survival. However, in the Danish study socioeconomic status did not affect the outcome. The Danish health care system is comparable to the Swedish system. Since no difference in overall survival between MIS and open surgery could be demonstrated and there are less complications in MIS, MIS should be advocated in elderly to facilitate and improve recovery.

Most endometrial cancers are diagnosed in an early stage and the RCTs included only presumed early FIGO stage patients. However, the finding of lymph node metastases render a higher final stage. In the RCT study by Walker at al., 14\% of patients were in FIGO stages IIIIV, and in this population-based cohort $11 \%$ were in stage III $(n=778)$. In some of the other RCTs the number of patients was very small in the advanced stages, so no comparisons were possible between MIS and open surgery. In our population cohort there was a high number of patients in stage III and we did not find any difference in survival outcome between MIS and open surgery in stage III (data not shown).

FIGO grade 3 endometrioid endometrial cancer is preoperatively classified as high-risk endometrial cancer since there is an increased risk for lymph node metastasis, therefore international and Swedish national guidelines recommend systematic pelvic and para-aortic lymph node dissection. In our material, FIGO grade 3 endometrioid carcinomas also showed significantly worse overall survival in the multivariable analysis.

The non-endometrioid carcinomas are known to have a worse prognosis than the endometrioid carcinomas, 
Table 4 Non-endometrioid endometrial carcinoma, FIGO stage I-III. Uni- and multivariable Cox proportional hazard regression analyzing overall survival including type of surgery, subtype, FIGO stage, and age

\begin{tabular}{|c|c|c|c|c|c|}
\hline \multirow[t]{2}{*}{ Variables } & \multirow[t]{2}{*}{$\begin{array}{l}\text { No. of } \\
\text { patients }\end{array}$} & \multicolumn{2}{|l|}{$\begin{array}{l}\text { Univariable } \\
\text { Cox regression }\end{array}$} & \multicolumn{2}{|l|}{$\begin{array}{l}\text { Multivariable } \\
\text { Cox regression }\end{array}$} \\
\hline & & HR (95\% Cl) & $p$ & HR (95\% Cl) & $p$ \\
\hline \multicolumn{6}{|l|}{ Surgical approach } \\
\hline MIS & 316 & Ref. & & Ref. & \\
\hline Open surgery & 573 & $1.10(0.86-1.42)$ & 0.44 & $0.98(0.76-1.27)$ & 0.88 \\
\hline \multicolumn{6}{|l|}{ Morphology ${ }^{a}$} \\
\hline Clear cell & 175 & $0.73(0.52-1.03)$ & 0.076 & $0.68(0.48-0.96)$ & 0.030 \\
\hline Serous & 495 & Ref. & & Ref. & \\
\hline Carcinosarcoma & 219 & $1.77(1.38-2.27)$ & $<0.001$ & $1.67(1.29-2.16)$ & $<0.001$ \\
\hline \multicolumn{6}{|l|}{ FIGO stage } \\
\hline IA & 372 & Ref. & & Ref. & \\
\hline IB & 143 & $2.26(1.54-3.32)$ & $<0.001$ & $1.99(1.34-2.93)$ & 0.001 \\
\hline$\|$ & 117 & $3.70(2.57-5.34)$ & $<0.001$ & $3.47(2.40-5.03)$ & $<0.001$ \\
\hline III & 257 & $4.50(3.30-6.12)$ & $<0.001$ & $4.68(3.42-6.40)$ & $<0.001$ \\
\hline \multicolumn{6}{|l|}{ Age group (years) } \\
\hline $0-59$ & 74 & Ref. & & Ref. & \\
\hline $60-69$ & 256 & $1.43(0.79-2.60)$ & 0.24 & $1.67(0.92-3.04)$ & 0.093 \\
\hline 70-79 & 359 & $2.18(1.23-3.87)$ & 0.008 & $2.50(1.41-4.46)$ & 0.002 \\
\hline 80- & 200 & $4.17(2.34-7.43)$ & $<0.001$ & $4.74(2.65-8.50)$ & $<0.001$ \\
\hline
\end{tabular}

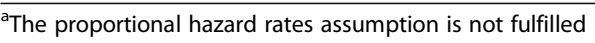

Table 5 Endometrial cancer, in patients with metastases in lymph nodes. Uni- and multivariable Cox proportional hazard regression analyzing overall survival including type of surgery, morphology, ploidy, lymphovascular space invasion and age

\begin{tabular}{|c|c|c|c|c|c|}
\hline \multirow[t]{2}{*}{ Variables } & \multirow[t]{2}{*}{$\begin{array}{l}\text { No. of } \\
\text { patients }\end{array}$} & \multicolumn{2}{|l|}{$\begin{array}{l}\text { Univariable } \\
\text { Cox regression }\end{array}$} & \multicolumn{2}{|l|}{$\begin{array}{l}\text { Multivariable } \\
\text { Cox regression }\end{array}$} \\
\hline & & HR $(95 \% \mathrm{Cl})$ & $p$ & HR $(95 \% \mathrm{Cl})$ & $p$ \\
\hline \multicolumn{6}{|l|}{ Surgical approach } \\
\hline MIS & 64 & Ref. & & Ref. & \\
\hline Open surgery & 137 & $1.31(0.70-2.45)$ & 0.40 & $1.36(0.72-2.57)$ & 0.34 \\
\hline \multicolumn{6}{|l|}{ Endometrioid } \\
\hline Yes & 132 & Ref. & & Ref. & \\
\hline No & 69 & $2.81(1.63-4.85)$ & $<0.001$ & $1.87(1.04-3.38)$ & 0.037 \\
\hline \multicolumn{6}{|l|}{ Ploidy } \\
\hline Diploid & 118 & Ref. & & Ref & \\
\hline Non-diploid & 83 & $2.65(1.36-5.14)$ & 0.004 & $2.19(1.09-4.40)$ & 0.029 \\
\hline \multicolumn{6}{|l|}{ LVSI } \\
\hline No & 61 & Ref. & & Ref. & \\
\hline Yes & 140 & $3.11(1.41-6.90)$ & 0.005 & $3.10(1.38-6.97)$ & 0.006 \\
\hline \multicolumn{6}{|l|}{ Age group (years) } \\
\hline $0-59$ & 43 & Ref. & & Ref. & \\
\hline $60-69$ & 72 & $1.28(0.53-3.11)$ & 0.59 & $1.33(0.55-3.24)$ & 0.53 \\
\hline $70-79$ & 68 & $2.84(1.22-6.60)$ & 0.015 & $2.60(1.11-6.12)$ & 0.028 \\
\hline $80-$ & 18 & $3.08(1.03-9.19)$ & 0.043 & $3.73(1.22-11.4)$ & 0.021 \\
\hline
\end{tabular}


Table 6 Endometrial cancer. Uni- and multivariable Cox proportional hazard regression analyzing overall survival including type of surgery, morphology, FIGO stage, ploidy, lymphovascular space invasion and age

\begin{tabular}{|c|c|c|c|c|c|}
\hline \multirow[t]{2}{*}{ Variables } & \multirow[t]{2}{*}{$\begin{array}{l}\text { No. of } \\
\text { patients }\end{array}$} & \multicolumn{2}{|l|}{$\begin{array}{l}\text { Univariable } \\
\text { Cox regression }\end{array}$} & \multicolumn{2}{|l|}{$\begin{array}{l}\text { Multivariable } \\
\text { Cox regression }\end{array}$} \\
\hline & & HR (95\% Cl) & $\mathrm{p}$ & $\mathrm{HR}(95 \% \mathrm{Cl})$ & $p$ \\
\hline \multicolumn{6}{|l|}{ Surgical approach } \\
\hline Robotic laparoscopy & 1986 & Ref. & & Ref. & \\
\hline Laparoscopy & 541 & $0.78(0.57-1.07)$ & 0.12 & $1.03(0.74-1.42)$ & 0.87 \\
\hline Open surgery & 2286 & $1.31(1.11-1.56)$ & 0.002 & $1.12(0.94-1.34)$ & 0.20 \\
\hline \multicolumn{6}{|l|}{ Endometrioid } \\
\hline Yes & 4378 & Ref. & & Ref. & \\
\hline No & 435 & $3.91(3.24-4.71)$ & $<0.001$ & $1.75(1.42-2.18)$ & $<0.001$ \\
\hline \multicolumn{6}{|l|}{ FIGO stage } \\
\hline IA & 2978 & Ref. & & Ref. & \\
\hline IB & 1019 & $2.09(1.69-2.58)$ & $<0.001$ & $1.32(1.06-1.66)$ & 0.013 \\
\hline$\|$ & 359 & $3.06(2.35-3.97)$ & $<0.001$ & $1.81(1.38-2.39)$ & $<0.001$ \\
\hline III & 457 & $6.25(5.10-7.67)$ & $<0.001$ & $2.75(2.16-3.49)$ & $<0.001$ \\
\hline \multicolumn{6}{|l|}{ Ploidy } \\
\hline Diploid & 3534 & Ref. & & Ref & \\
\hline Non-diploid & 1279 & $2.75(2.34-3.23)$ & $<0.001$ & $1.59(1.33-1.91)$ & $<0.001$ \\
\hline \multicolumn{6}{|l|}{ LVSI $^{a}$} \\
\hline No & 3896 & Ref. & & Ref. & \\
\hline Yes & 917 & $4.24(3.61-4.98)$ & $<0.001$ & $2.25(1.86-2.72)$ & $<0.001$ \\
\hline \multicolumn{6}{|l|}{ Age group (years) } \\
\hline $0-59$ & 943 & Ref. & & Ref. & \\
\hline $60-69$ & 1535 & $2.11(1.44-3.10)$ & $<0.001$ & $1.88(1.28-2.76)$ & 0.001 \\
\hline $70-79$ & 1624 & $4.40(3.07-6.31)$ & $<0.001$ & $3.66(2.55-5.26)$ & $<0.001$ \\
\hline 80- & 711 & $10.7(7.44-15.3)$ & $<0.001$ & $8.65(6.00-12.4)$ & $<0.001$ \\
\hline
\end{tabular}

${ }^{\text {a }}$ The proportional hazard rates assumption is not fulfilled

therefore in most guidelines they are classified as highrisk and adjuvant therapy with chemo- +/- radiotherapy is recommended [21]. The non-endometrioid carcinomas are often included in randomized controlled endometrial cancer studies even though it is often not possible to analyze the subtypes separately due to their low number [7]. This large study included 924 patients with non-endometrioid carcinomas, MIS and laparotomy showed no difference in the multivariable analysis. Carcinosarcoma showed the worst prognosis similar to a smaller Canadian study [22]. Clear cell carcinomas showed the best prognosis of the non-endometrioid tumors in line with an American study [23].

Our group previously reported LVSI as an independent risk factor for lymph node metastases and decreased survival in patients with endometrioid adenocarcinomas [24]. In this material we also included the nonendometrioid carcinomas where, in the multivariable analyses, we found LVSI to be an independent prognostic factor for decreased overall survival in endometrial cancer stage I-III. Available evidence suggests that LVSI in the primary tumor may serve as a marker for both lymphatic and hematologic dissemination $[25,26]$.

This nationwide population-based registry study using prospectively collected data may include selection bias. Women with a large uterus that may contain a larger tumor as well as obvious enlarged lymph nodes on CT scan may have been selected for open surgery. The health care system is relatively uniform all over Sweden and is free of charge to all citizens living in Sweden. The studies in the Cochrane meta-analysis included five RCTs with 3993 patients in the primary outcome overall survival and only one study finished after 2013 [20]. Our single registry study had no exclusion criteria, almost twice as many patients as the Cochrane meta-analysis and was run in a population-based manner including all patients in the regular national health care system. In Sweden almost no cancer patients receive treatment by private health care providers. Large register studies such as the present and the Danish study by Jorgensen et al. 
[15] including confounders in the multivariable analyses add knowledge how the surgical or any treatment modality work in populations in the regular health care system.

There are some limitations to be considered e.g. that the Swedish Guidelines for Endometrial cancer published in 2011 included recommendations for lymph node staging and the procedure was gradually implemented, which is why not all grade 3 endometrioid endometrial cancer patients or patients with high-risk histology had a lymphadenectomy performed during the beginning of the study period, perhaps leading to under-staging. Moreover, not all patients had LVSI analyzed. The registry includes no molecular analyses which should be considered in the future. However, the number of patients with LVSI analyzed was high: 4813 in the multivariable analyses. Robotassisted surgery has been introduced the last 10 years and it is performed mostly at tertiary centers by experienced surgeons. This may have improved the outcome in the MIS group. During the last 2 years of the study period the sentinel lymph node concept was introduced, and some university centers also used this technique in low-risk endometrial cancer patients. For all patients with high-risk endometrial cancer, however, the recommendation of full lymph node staging up to the renal veins remained. It is unknown how sentinel lymph node mapping will impact patient outcomes, including the need for adjuvant postoperative radio- and/or chemotherapy, as well as the incidence of adverse events and survival. Future analyses concerning oncological outcomes after the implementation of sentinel lymph node procedures are needed to evaluate the long-term effects.

\section{Conclusion}

In this large population-based study including over 7000 patients with endometrial cancer stages I-III, surgical approach, MIS or open surgery, had no influence on overall survival when adjustment was made for known prognostic factors.

\footnotetext{
Abbreviations

FIGO: The Federation Internationale de Gynecologie et d'Obstetrique: MIS: Minimally invasive surgery; NCR: Swedish National Cancer Registry; SQRG C: The Swedish Quality Registry for Gynecologic Cancer;

LVSI: Lymphovascular space involvement; Cl: Confidence intervals; WHO: World Health Organization; OS: Overall survival; DNA: Deoxyribonucleic acid; US: United States of America; RCT: Randomized controlled trial
}

\section{Supplementary Information}

The online version contains supplementary material available at https://doi. org/10.1186/s12885-021-08289-3.

Additional file 1: Supplementary Table 1. Endometrial cancer. Uniand multivariable Cox proportional hazard regression analyzing overall survival including type of surgery, morphology, lymph node metastases, ploidy, lymphovascular space invasion and age.
Additional file 2: Supplementary Table 2. Separate uni- and multivariable Cox proportional hazard regression analyses for the specific histologies/morphologies performed for each morphology.

\section{Acknowledgements}

We thank the Swedish Quality Registry for Gynecologic Cancer (SQRGC) as well as the Swedish Association of Local Authorities and Regions (SKR), which financially supports the Swedish Quality Registry for Gynecologic Cancer (SQRGC). This work was also supported by grants from the Swedish Cancer Society and the Regional founds in Region Skåne.

\section{Authors' contributions}

All authors contributed to the study plan. CB performed conceptualization, manuscript writing, reviewing, and editing. $\mathrm{EH} 1$ did the statistical analyzes, manuscript writing, reviewing, and editing. JM, KS, BT, EÅL, AFR, MB, PDK, KH, $\mathrm{EH} 2, \mathrm{PK}$, and PR were critically revising the manuscript. TH was critically revising and editing the manuscript. All authors have read and approved the manuscript.

\section{Funding}

The Swedish Association of Local Authorities and Regions (SKR), financially supports the Swedish Quality Registry for Gynecologic Cancer (SQRGC). This work was also supported by grants from the Swedish Cancer Society and the Regional founds in Region Skåne which provided resources to plan the study, to collect and analyze the data and to write the report. The funders did not have any impact on the study design, choice of methods, collection of data, analyses or on the conclusions drawn. Open access funding was provided by Lund University.

\section{Availability of data and materials}

All data and the material are available at the Regional Cancer Center West where the Swedish Quality Registry for Gynecologic Cancer (SQRGC) is administrated and located.

\section{Declarations}

\section{Ethics approval and consent to participate}

The ethical review board at Gothenburg University approved the study (Dnr 814-15). According to Swedish legislation, patients should be informed at admission to the clinical department that they will be registered in a quality register. No signed consent is necessary, but the patient can negate participation in the register.

\section{Consent for publication}

All the authors have read the final manuscript and consent the manuscript for publication.

\section{Competing interests}

No conflicts of interest have been declared by any of the authors.

\section{Author details}

${ }^{1}$ Department of Obstetrics and Gynecology, Skåne University Hospital, Lund University, SE-22185 Lund, Sweden. ${ }^{2}$ Region Västra Götaland, Regional Cancer Centre West, SE-41345 Gothenburg, Sweden. ${ }^{3}$ Department of Obstetrics and Gynecology, Halland Hospital, SE-43281 Varberg, Sweden. ${ }^{4}$ Department of Women's and Children's Health, Uppsala University, SE-75185 Uppsala, Sweden. ${ }^{5}$ Department of Oncology, Uppsala University Hospital, SE-75185 Uppsala, Sweden. ${ }^{6}$ Department of Oncology and Department of Biomedical and Clinical Sciences, Linköping University, SE-58185 Linköping, Sweden. ${ }^{7}$ Department of Women's and Children's Health, Division of Neonatology, Obstetrics and Gynecology, Karolinska Institutet, Karolinska University Hospital, SE-17176 Stockholm, Sweden. ${ }^{8}$ Department of Hematology, Oncology and Radiation Physics, Skåne University Hospital, and Department of Clinical Sciences, Lund University, SE-22185 Lund, Sweden. 'Department of Obstetrics and Gynecology, Institute of Clinical Sciences, Sahlgrenska Academy, SE-41345 Gothenburg, Sweden. ${ }^{10}$ Department of Gynecologic Cancer, Theme Cancer, Karolinska University Hospital, SE-171 76 Stockholm, Sweden. ${ }^{11}$ Department of Gynecology and Obstetrics, Visby Hospital, SE-62155 Visby, Sweden. ${ }^{12}$ Department of Obstetrics and Gynecology in Linköping, Department of Biomedical and Clinical Sciences, Linköping 
University, SE-58185 Linköping, Sweden. ${ }^{13}$ Department of Medical Oncology, Department of Clinical Sciences, Lund University, SE-22100 Lund, Sweden.

Received: 31 October 2020 Accepted: 4 May 2021

Published online: 02 June 2021

\section{References}

1. Amant F, Moerman P, Neven P, Timmerman D, Van Limbergen E, Vergote I. Endometrial cancer. Lancet (London, England). 2005;366(9484):491-505.

2. Felix AS, Bower JK, Pfeiffer RM, Raman SV, Cohn DE, Sherman ME. High cardiovascular disease mortality after endometrial cancer diagnosis: results from the surveillance, epidemiology, and end results (SEER) database. Int J Cancer. 2017;140(3):555-64. https://doi.org/10.1002/ijc.30470.

3. Borgfeldt C, Kalapotharakos G, Asciutto KC, Lofgren M, Hogberg T. A population-based registry study evaluating surgery in newly diagnosed uterine cancer. Acta Obstet Gynecol Scand. 2016;95(8):901-11. https://doi. org/10.1111/aogs.12918.

4. Ran L, Jin J, Xu Y, Bu Y, Song F. Comparison of robotic surgery with laparoscopy and laparotomy for treatment of endometrial cancer: a metaanalysis. PLoS One. 2014;9(9):e108361. https://doi.org/10.1371/journal.pone. 0108361.

5. Salehi S, Avall-Lundqvist E, Legerstam B, Carlson JW, Falconer H. Robotassisted laparoscopy versus laparotomy for infrarenal paraaortic lymphadenectomy in women with high-risk endometrial cancer: a randomised controlled trial. Eur J Cancer. 2017;79:81-9. https://doi.org/10.1 016/j.ejca.2017.03.038.

6. Lindfors A, Akesson A, Staf C, Sjoli P, Sundfeldt K, Dahm-Kahler P. Robotic vs open surgery for endometrial Cancer in elderly patients: surgical outcome, survival, and cost analysis. Int J Gynecol Cancer. 2018;28(4):692-9. https:// doi.org/10.1097/IGC.0000000000001240.

7. Walker UL, Piedmonte MR, Spirtos NM, Eisenkop SM, Schlaerth JB, Mannel RS, et al. Recurrence and survival after random assignment to laparoscopy versus laparotomy for comprehensive surgical staging of uterine cancer: gynecologic oncology group LAP2 study. J Clin Oncol. 2012;30(7):695-700. https://doi.org/10.1200/JCO.2011.38.8645.

8. Janda M, Gebski V, Davies LC, Forder P, Brand A, Hogg R, et al. Effect of Total laparoscopic hysterectomy vs Total abdominal hysterectomy on disease-free survival among women with stage I endometrial Cancer: a randomized clinical trial. JAMA. 2017;317(12):1224-33. https://doi.org/10.1 001/jama.2017.2068.

9. Ramirez PT, Frumovitz M, Pareja R, Lopez A, Vieira M, Ribeiro R, et al. Minimally invasive versus abdominal radical hysterectomy for cervical Cancer. N Engl J Med. 2018;379(20):1895-904. https://doi.org/10.1056/ NEJMoa1806395.

10. Melamed A, Margul DJ, Chen L, Keating NL, del Carmen MG, Yang J, et al. Survival after minimally invasive radical hysterectomy for early-stage cervical Cancer. N Engl J Med. 2018;379(20):1905-14. https://doi.org/10.1056/ NEJMoa1804923.

11. Rosenberg P, Kjolhede P, Staf C, et al. Data quality in the Swedish quality register of gynecologic Cancer - a Swedish gynecologic Cancer group (SweGCG) study. Acta Oncol. 2018;57(3):346-53. https://doi.org/10.1080/02 84186X.2017.1366048.

12. Pecorelli S. Revised FIGO staging for carcinoma of the vulva, cervix, and endometrium. Int J Gynaecol Obstet. 2009;105(2):103-4. https://doi.org/10.1 016/j.jigo.2009.02.012.

13. Baldetorp B, Dalberg M, Holst U, Lindgren G. Statistical evaluation of cell kinetic data from DNA flow cytometry (FCM) by the EM algorithm. Cytometry. 1989;10(6):695-705. https://doi.org/10.1002/cyto.990100605.

14. Auer G, Askensten U, Ahrens O. Cytophotometry. Hum Pathol. 1989;20(6): 518-27. https://doi.org/10.1016/0046-8177(89)90243-8.

15. Jorgensen SL, Mogensen O, Wu CS, Korsholm M, Lund K, Jensen PT. Survival after a nationwide introduction of robotic surgery in women with earlystage endometrial cancer: a population-based prospective cohort study. Eur J Cancer. 2019;109:1-11. https://doi.org/10.1016/j.ejca.2018.12.004.

16. Sandadi S, Gadzinski JA, Lee S, Chi DS, Sonoda Y, Jewell EL, et al. Fellowship learning curve associated with completing a robotic assisted total laparoscopic hysterectomy. Gynecol Oncol. 2014;132(1):102-6. https://doi. org/10.1016/j.ygyno.2013.11.017.

17. Seamon LG, Fowler JM, Richardson DL, Carlson MJ, Valmadre S, Phillips GS, et al. A detailed analysis of the learning curve: robotic hysterectomy and pelvic-aortic lymphadenectomy for endometrial cancer. Gynecol Oncol. 2009;114(2):162-7. https://doi.org/10.1016/j.ygyno.2009.04.017.

18. Bergstrom J, Aloisi A, Armbruster S, Yen TT, Casarin J, Leitao MM Jr, et al. Minimally invasive hysterectomy surgery rates for endometrial cancer performed at National Comprehensive Cancer Network (NCCN) centers. Gynecol Oncol. 2018;148(3):480-4. https://doi.org/10.1016/j. ygyno.2018.01.002.

19. Wright JD, Burke WM, Tergas Al, Hou JY, Huang Y, Hu JC, et al. Comparative effectiveness of minimally invasive hysterectomy for endometrial Cancer. J Clin Oncol. 2016;34(10):1087-96. https://doi.org/10.1200/JCO.2015.65.3212.

20. Galaal K, Donkers H, Bryant A, Lopes AD. Laparoscopy versus laparotomy for the management of early stage endometrial cancer. Cochrane Database Syst Rev. 2018;10:CD006655.

21. Morice P, Leary A, Creutzberg C, Abu-Rustum N, Darai E. Endometrial cancer Lancet (London, England). 2016;387(10023):1094-108.

22. Altman AD, Ferguson SE, Atenafu EG, Köbel M, McAlpine JN, Panzarella T, et al. Canadian high risk endometrial cancer (CHREC) consortium: analyzing the clinical behavior of high risk endometrial cancers. Gynecol Oncol. 2015; 139(2):268-74. https://doi.org/10.1016/j.ygyno.2015.09.001.

23. Hamilton CA, Cheung MK, Osann K, Chen L, Teng NN, Longacre TA, et al. Uterine papillary serous and clear cell carcinomas predict for poorer survival compared to grade 3 endometrioid corpus cancers. Br J Cancer. 2006;94(5): 642-6. https://doi.org/10.1038/sj.bjc.6603012.

24. Stalberg K, Bjurberg M, Borgfeldt C, et al. Lymphovascular space invasion as a predictive factor for lymph node metastases and survival in endometrioid endometrial cancer - a Swedish gynecologic Cancer group (SweGCG) study. Acta Oncol. 2019;58(11):1628-33. https://doi.org/10.1080/0284186X.2019.1 643036

25. Bosse T, Peters EE, Creutzberg CL, et al. Substantial lymph-vascular space invasion (LVSI) is a significant risk factor for recurrence in endometrial cancer--a pooled analysis of PORTEC 1 and 2 trials. Eur J Cancer. 2015;51(13): 1742-50. https://doi.org/10.1016/j.ejca.2015.05.015.

26. Sato M, Taguchi A, Fukui Y, Kawata A, Taguchi S, Kashiyama T, et al. Blood vessel invasion is a strong predictor of postoperative recurrence in endometrial Cancer. Int J Gynecol Cancer. 2018;28(5):875-81. https://doi. org/10.1097//GC.0000000000001262.

\section{Publisher's Note}

Springer Nature remains neutral with regard to jurisdictional claims in published maps and institutional affiliations.
Ready to submit your research? Choose BMC and benefit from:

- fast, convenient online submission

- thorough peer review by experienced researchers in your field

- rapid publication on acceptance

- support for research data, including large and complex data types

- gold Open Access which fosters wider collaboration and increased citations

- maximum visibility for your research: over $100 \mathrm{M}$ website views per year

At $\mathrm{BMC}$, research is always in progress.

Learn more biomedcentral.com/submissions 